\title{
NORMAL CONTROL PROBLEMS HAVE NO MINIMIZING STRICTLY ORIGINAL SOLUTIONS ${ }^{1}$
}

\author{
BY J. WARGA
}

Communicated by Victor Klee, February 12, 1971

\begin{abstract}
We prove for a general optimal control problem that, in the absence of abnormal admissible extremals (solutions of a generalized Weierstrass E-condition), any control which is optimal in the set of original (ordinary) controls must also be optimal in the larger set of relaxed (measure-valued) controls.
\end{abstract}

1. We consider the model of an optimal control problem studied in [2]. This model was found applicable, among others, to unilateral control problems defined by ordinary differential and multidimensional integral equations [3], evasion problems [4], and conflicting control problems [5]. For the sake of completeness, we begin by restating the definition of this model. Let $T$ and $R$ be compact metric spaces and $\mu$ a positive and nonatomic Radon measure on $T$. We denote by $\operatorname{rpm}(R)$ the set of regular Borel probability measures on $R$ endowed with the relative weak star topology of $C(R)^{*}$, by $a$ the class of $\mu$-measurable functions on $T$ to $R$ (original control functions), and by $\delta$ the set of $\mu$-measurable functions on $T$ to $\operatorname{rpm}(R)$ (relaxed control functions). We embed $R$ in $\operatorname{rpm}(R)$ and $R$ in $\delta$ by identifying each $r \in R$ with the Dirac measure at $r$. In turn, we view $S$ as a subset of $L^{1}(T, C(R))^{*}$, and endow it with the relative weak star topology, by identifying each $\sigma \in \mathcal{S}$ with the functional $\phi \rightarrow \int \mu(d t) \int \phi(t)(r) \sigma(t)(d r)$.

Now let $R$ be the real line, $X$ a real topological vector space, $C$ a convex body in $X, B$ a convex subset of a vector space (the set of control parameters $), m$ a positive integer, $x=\left(x_{0}, x_{1}, x_{2}\right): s \times B \rightarrow R \times R^{m}$ $\times x$ a given function, and

$$
a(\mathcal{U})=\left\{(\sigma, b) \in \mathcal{u} \times B \mid x_{1}(\sigma, b)=0, x_{2}(\sigma, b) \in C\right\} \quad(\mathcal{} \subset s) .
$$

We say that $(\bar{\sigma}, \bar{b})$ is a minimizing original (respectively relaxed) solution if it yields a minimum of $x_{0}$ on $Q(a)$ (respectively on $Q(S)$ ). A minimizing original solution is a minimizing strictly original solution if it is not at the same time a minimizing relaxed solution. We set $Q=\S \times B$, denote by $\Im_{m+1}$ the simplex $\left\{\left(\theta^{0}, \cdots, \theta^{m}\right) \in R^{m+1} \mid \theta^{j} \geqq 0\right.$,

AMS 1970 subject classifications. Primary 49A25, 49B30; Secondary 49A50.

Key words and phrases. Model, optimal control problem, minimizing original solution, minimizing relaxed solution, abnormal extremal.

1 Partly supported by N.A.S.A. grant NGR 22-011-020. 
$\left.\sum_{j=0}^{m} \theta^{j} \leqq 1\right\}$ and by $D x(\tilde{q} ; q-\tilde{q})$ the directional derivative $\lim _{\alpha \rightarrow+0} \alpha^{-1}[x(\tilde{q}+\alpha(q-\tilde{q}))-x(\tilde{q})]$. For $\tilde{q}, q_{0}, q_{1}, \cdots, q_{m} \in Q$, we say that the function

$$
\theta \rightarrow x\left(\tilde{q}+\sum_{j=0}^{m} \theta^{j}\left(q_{j}-\tilde{q}\right)\right): I_{m+1} \rightarrow R \times R^{m} \times x
$$

is differentiable at 0 if it has a Fréchet derivative at 0 relative to $\Im_{m+1}$, i.e. if

$$
\lim |\theta|^{-1}\left[x\left(\tilde{q}+\sum_{j=0}^{m} \theta^{j}\left(q_{j}-\tilde{q}\right)\right)-x(q)-\sum_{j=0}^{m} \theta^{j} D x\left(\tilde{q} ; q_{j}-\tilde{q}\right)\right]=0
$$

in $R \times R^{m} \times \mathscr{X}$ as $|\theta| \rightarrow 0, \theta \in J_{m+1}$.

Points $\tilde{q}=(\tilde{\sigma}, \tilde{b}) \in \mathcal{S} \times B$ and $l=\left(l_{0}, l_{1}, l_{2}\right) \in[0, \infty) \times R^{m} \times x^{*}$ define an extremal $(\tilde{q}, l)$, and $\tilde{q}$ is extremal if $\tilde{q}$ and $l$ satisfy the generalized Weierstrass E-condition (maximum principle)

$l \neq 0, \quad l(D x(\tilde{q} ; q-\tilde{q})) \geqq 0 \quad(q \in Q) \quad$ and $\quad l_{2}\left(x_{2}(\tilde{q})\right) \geqq l(c) \quad(c \in C)$.

An extremal $(\tilde{q}, l)$ is admissible if $\tilde{q}=(\tilde{\sigma}, \tilde{b}) \in Q(S)$; an extremal $(\tilde{q}, l)$ $=\left(\tilde{q}, l_{0}, l_{1}, l_{2}\right)$ is abnormal if $l_{0}=0$. The optimal control problem is normal if there exist no abnormal admissible extremals.

Theorem I. Assume that, for each choice of $\tilde{q}, q_{0}, \cdots, q_{m} \in Q$, with $\tilde{q}=(\tilde{\sigma}, \tilde{b})$ and $q_{i}=\left(\sigma_{i}, b_{i}\right)(i=0,1, \cdots, m)$, the function

$$
(\sigma, \theta) \rightarrow x\left(\sigma, \tilde{b}+\sum_{j=0}^{m} \theta^{j}\left(b_{j}-\tilde{b}\right)\right): \delta \times J_{m+1} \rightarrow R \times R^{m} \times x
$$

is continuous and the function

$$
\theta \rightarrow x\left(\tilde{q}+\sum_{j=0}^{m} \theta^{j}\left(q_{j}-\tilde{q}\right)\right): \Im_{m+1} \rightarrow R \times R^{m} \times x
$$

is differentiable at 0 . If $(\bar{\rho}, \bar{b})$ is a minimizing strictly original solution then there exists an abnormal admissible extremal $\left(\sigma^{\#}, b^{\#}, 0, l_{1}, l_{2}\right)$ such that $x_{0}\left(\sigma^{\sharp}, b^{\sharp}\right)<x_{0}(\bar{\rho}, \bar{b})$.

Proof. Let $(\bar{\rho}, \bar{b})$ be a minimizing strictly original solution. We set

$$
\begin{gathered}
B^{\prime}=B \times R, \quad x^{\prime}=x \times R, \quad C^{\prime}=C \times(-\infty, 0), \\
x_{0}^{\prime}\left(\sigma, b^{\prime}\right)=\alpha, \quad x_{1}^{\prime}\left(\sigma, b^{\prime}\right)=x_{1}(\sigma, b), \\
x_{2}^{\prime}\left(\sigma, b^{\prime}\right)=\left(x_{2}(\sigma, b), x_{0}(\sigma, b)-x_{0}(\bar{\rho}, \bar{b})\right) \quad\left(\sigma \in \delta, b^{\prime}=(b, \alpha) \in B^{\prime}\right), \\
x^{\prime}=\left(x_{0}^{\prime}, x_{1}^{\prime}, x_{2}^{\prime}\right) .
\end{gathered}
$$


We denote by $P$ the optimal control problem we are considering and by $P^{\prime}$ the problem obtained by replacing $B, x, C$ and $x$ with $B^{\prime}, x^{\prime}$, $C^{\prime}$ and $x^{\prime}$, respectively. Since $(\bar{\rho}, \bar{b})$ does not minimize $x_{0}$ on $Q(\delta)$, there exists $\left(\sigma^{\sharp}, b^{\sharp}\right) \in Q(S)$ such that $x_{0}\left(\sigma^{\sharp}, b^{\#}\right)<x_{0}(\bar{\rho}, \bar{b})$. It follows that $x_{1}^{\prime}\left(\sigma^{\sharp}, b^{\sharp}, 0\right)=0$ and $x_{2}^{\prime}\left(\sigma^{\sharp}, b^{\sharp}, 0\right) \in C^{\prime}$. The argument of $[2,4.1$, Proof of Theorem 2.2, p. 369], when applied to $P^{\prime}$ and $\left(\sigma^{*}, b^{\#}, 0\right)$, shows that either (a) there exists $l=\left(l_{0}, l_{1}, l_{2}^{\prime}\right) \in[0, \infty) \times R^{m} \times(x \times R)^{*}$ such that $\left(\sigma^{\#}, b^{\#}, 0, l_{0}, l_{1}, l_{2}^{\prime}\right)$ is an admissible extremal of $P^{\prime}$, or (b) there exists $\left(\rho_{1}, b_{1}, \alpha_{1}\right) \in R \times B^{\prime}$ such that $x_{1}^{\prime}\left(\rho_{1}, b_{1}, \alpha_{1}\right)=x_{1}\left(\rho_{1}, b_{1}\right)=0$ and $x_{2}^{\prime}\left(\rho_{1}, b_{1}, \alpha_{1}\right) \in C^{\prime}$; hence $x_{2}\left(\rho_{1}, b_{1}\right) \in C$ and $x_{0}\left(\rho_{1}, b_{1}\right)<x_{0}(\bar{\rho}, \bar{b})$. The alternative (b) must be discarded because it conflicts with the assumption that $(\bar{\rho}, \bar{b})$ is a minimizing original solution. We set, in (a), $l_{2}^{\prime}=\left(l_{2}, \lambda_{0}\right) \in x^{*} \times R$ and $q^{\#}=\left(\sigma^{\#}, b^{\#}\right)$, and conclude that

$$
\begin{array}{r}
l \neq 0, \quad l_{0} \alpha+l_{1} D x_{1}\left(q^{\#} ; q-q^{\#}\right)+l_{2}\left(D x_{2}\left(q^{\#} ; q-q^{\#}\right)\right)+\lambda_{0} D x_{0}\left(q^{\#} ; q-q^{\#}\right) \geqq 0 \\
(\alpha \in R, q=(\sigma, b) \in \S \times B)
\end{array}
$$

and

$l_{2}\left(x_{2}\left(q^{\#}\right)\right)+\lambda_{0}\left[x_{0}\left(q^{\#}\right)-x_{0}(\bar{\rho}, \bar{b})\right] \geqq l_{2}(c)+\lambda_{0} \alpha \quad(c \in C, \alpha \in(-\infty, 0))$.

Since $x_{0}\left(q^{\#}\right)<x_{0}(\bar{\rho}, \bar{b})$, these relations imply that $\lambda_{0}=l_{0}=0$ and show that $\left(\sigma^{\#}, b^{\#}, 0, l_{1}, l_{2}\right)$ is an abnormal admissible extremal of $P$. Q.E.D.

2. Theorem I can be applied, under certain conditions, to problems where the original control functions are not a priori restricted to a compact set (e.g. to a problem of Bolza when its admissible extremals have uniformly bounded derivatives). Examples can be given [6, p. 118] of simple problems that possess minimizing strictly original solutions but, in view of Theorem I, these problems cannot be normal. If we add (to those of Theorem I) the assumptions that $Q(\S)$ is nonempty and there exists a sequentially compact topology of $B$ such that $x$ is continuous on $\mathcal{S} \times B$ (or an appropriate subset) then, by [2, Theorems 2.1 and 2.2, pp. 362-363], there exists a minimizing relaxed solution and it is extremal. Thus, in normal problems, a minimizing original solution exists if and only if there exists an extremal point $(\bar{\rho}, \bar{b}) \in \mathbb{Q}(\Omega)$ that minimizes $x_{0}$ among all extremal $(\tilde{\sigma}, \tilde{b}) \in Q(S)$. This suggests that the most promising approach to a theory of minimizing original solutions will remain the one that led to the justification of the Dirichlet principle and that McShane [1] applied in 1940 to the Bolza problem (using Young's [7], [8] generalized curves as tools); namely, the investigation of conditions insuring that weak solutions of the problem (such as minimizing gen- 
eralized curves or minimizing relaxed solutions) are also "classical" solutions.

We expect to publish elsewhere extensions of Theorem I with somewhat weaker hypotheses and with original control functions restricted by the condition $\rho(t) \in R^{\sharp}(t) \mu$-a.e., where $R^{\sharp}(\cdot)$ is a given $\mu$-measurable set-valued mapping. We shall also demonstrate the applicability of the model to functional-integral equations in $C\left(T, R^{n}\right)$ and $L^{p}\left(T, R^{n}\right)$.

\section{REFERENCES}

1. E. J. McShane, Existence theorems for Bolza problems in the calculus of variations, Duke Math. J. 7 (1940), 28-61. MR 2, 226.

2. J. Warga, Control problems with functional restrictions, SIAM J. Control 8 (1970), 360-371.

3. - - Unilateral and minimax control problems defined by integral equations, SIAM J. Control 8 (1970), 372-382.

4. - On a class of pursuit and evasion problems, J. Differential Equations 9 (1971), 155-167. 673.

5. - Conflicting and minimax controls, J. Math. Anal. Appl. 33 (1971), 655-

6. —_. Relaxed variational problems, J. Math. Anal. Appl. 4 (1962), 111-128. MR $25 \# 54152$.

7. L. C. Young, Generalized curves and the existence of an attained absolute minimum in the calculus of variations, C. R. Soc. Sci. Lett. Varsovie Cl. III. 30 (1937), 212234.

8. - - Necessary conditions in the calculus of variations, Acta Math. 69 (1938), 239-258.

Northeastern University, Boston, Massachusetts 02115 